\title{
Spéciation de l'arsenic par couplage LC-ICP-MS : avantages et inconvénients en biologie médicale
}

\section{Arsenic speciation by LC-ICP-MS : advanta- ges and disadvantages in clinical chemistry}

Anne LE BOUIL

Service de Pharmacologie et Toxicologie, CHU d'Angers.

*Auteur à qui adresser la correspondance : Anne LE BOUIL, Service de Pharmacologie et Toxicologie,

CHU d'Angers, 4, rue Larrey, 49933 ANGERS cedex 9

Tél : 0241353644 - Fax : 0241354877 - E-mail : anlebouil@chu-angers.fr

(Reçu le 8 février 2007 ; accepté après modifications le 15 avril 2007)

\section{RÉSUMÉ}

La toxicité de l'arsenic dépendant de la forme chimique sous laquelle il se trouve, le dosage de l'arsenic total seul ne permet pas une interprétation correcte en biologie médicale. Il peut, tout au plus, être utilisé comme analyse de dépistage afin d'écarter une éventuelle intoxication. La spéciation s'impose. De nombreuses techniques permettent aujourd'hui de quantifier les différentes espèces d'arsenic. Le couplage de la chromatographie en phase liquide à la spectrométrie de masse couplée à un plasma induit par haute fréquence ( $L C$ ICP-MS) est l'une des plus utilisées.

L'avantage principal de ce couplage réside dans les qualités de l'ICP-MS en tant que détecteur, à savoir sensibilité, spécificité, gamme de mesure étendue (dynamic range). Les limites de détection obtenues en LC-ICP-MS varient selon les auteurs et les formes d'arsenic de 0,5 $\mu \mathrm{g} / \mathrm{L}$ à $10 \mu \mathrm{g} / \mathrm{L}$ dans l'urine, ce qui permet d'atteindre des niveaux de concentration rencontrés chez les sujets non exposés. L'autre avantage est la facilité dans la mise en æuvre du couplage entre le système chromatographique et l'ICP-MS. En effet, il suffit de raccorder la sortie de la colonne par un tuyau (type tube en PEEK®) au

\section{SUMMARY}

The toxicity of arsenic depends on its chemical form, therefore determination of total arsenic concentration is not sufficient in clinical chemistry. Total arsenic determination can be used as a screening test to rule out an intoxication. It is necessary to measure individual concentration of every arsenical species. Today, arsenic speciation can be carried out using several hyphenated techniques. High performance liquid chromatography coupled with inductively coupled plasma mass spectrometry (LC-ICP-MS) is one of the most commonly used methods. The principal advantages of this method are the qualities of ICP-MS : high sensitivity, large dynamic range and selectivity. The detection limits achieved by LC-ICP-MS are between 0,5 and $10 \mu \mathrm{g} / \mathrm{L}$ according to the arsenical species and the authors, which permits the speciation in biological samples from unexposed persons. Another advantage is the simplicity of the interface. The outlet from the HPLC column is directly connected to the ICP-MS nebulizer with a capillary tube (PEEK ${ }^{\circledR}$ tubing). Usual chromatographic flows $(\approx 1 \mathrm{ml} / \mathrm{min})$ are compatible with the nebulizer uptake rate of the ICP-MS instrument. The main inconvenience is the cost of investment and running. The consumption of argon 
nébuliseur de la torche. Les débits classiquement utilisés en chromatographie liquide $(\approx 1 \mathrm{ml} / \mathrm{min})$ sont compatibles avec le fonctionnement de la torche à plasma.

Les inconvénients sont d'ordre économique et chromatographique. Le coût d'achat et de fonctionnement lié à la consommation d'argon $(15 \mathrm{~L} / \mathrm{min})$ n'est pas à la portée de tous les laboratoires. La consommation d'argon est un paramètre à prendre en compte en LC-ICP-MS car si la durée moyenne d'une analyse en mode de fonctionnement ICP-MS normal est de deux minutes, la durée de l'analyse chromatographique est plutôt de l'ordre d'une dizaine de minutes. L'utilisation de l'ICP-MS comme détecteur impose des contraintes dans le choix de la phase mobile. Une quantité trop importante de solvant organique déstabilise le plasma, limitant celle-ci à des concentrations généralement inférieures à $5 \%$. D'autre part, des concentrations de tampon supérieures à $50 \mathrm{mM}$ peuvent être à l'origine de dépôts de sels au niveau du nébuliseur et du cône échantillonneur.

En conclusion, si la LC-ICP-MS est une technique répondant aux besoins en biologie médicale en termes de performances analytiques, elle n'en demeure pas moins une technique coûteuse.

\section{MOTS-CLÉS}

Arsenic, spéciation, LC-ICP-MS.

\section{Introduction}

L'arsenic est présent dans l'environnement sous différentes formes chimiques dont la toxicité varie beaucoup (Tableau I) (1). L'arsenic inorganique (Asi) sous forme d'arsénite $\left(\mathrm{As}^{\mathrm{III}}\right)$ et d'arséniate $\left(\mathrm{As}^{\mathrm{V}}\right)$, est le plus toxique. Chez l'homme, l'arsenic inorganique est métabolisé et excrété dans l'urine sous forme de dérivés méthylés pentavalents moins toxiques : l'acide monométhylarsonique $\left(\mathrm{MMA}^{\mathrm{v}}\right)$ et l'acide diméthylarsinique $\left(\mathrm{DMA}^{\mathrm{v}}\right)$. La biométhylation de l'arsenic met en jeu successivement une réduction de l'arsenic pentavalent puis une méthylation oxydative avec la S-adénosylméthionine comme donneur du groupement méthyle. Au cours de cette étape, des intermédiaires méthylés trivalents très réactifs, $\mathrm{MMA}^{\mathrm{III}}$ et DMA ${ }^{\mathrm{III}}$, sont formés (2).

L'arsenic contenu dans les produits de la mer sous forme majoritairement d'arsénobétaïne (AsB) et en moins grande quantité d'arsénocholine et de sucres arséniés n'est pas toxique. L'arsénobétaïne, après ingestion, est rapidement éliminé dans l'urine sans biotransformation.

De nos jours, les intoxications aiguës massives à l'arsenic minéral sont rares du fait de la limitation de son usage en thérapeutique et en agriculture. Des intoxications chroniques peuvent se rencontrer dans un cadre professionnel (métallurgie, industrie du verre, fabrication de pigments et préservation du bois) ou dans certains pays par une eau de boisson contaminée (en
(15 L/min) must be taken into account because the times of analysis by LC-ICP-MS are often more than ten minutes. The use of ICP-MS imposes some limitations on the composition of the mobile phase. The introduction of organic solvents can destabilize the plasma, so only percentages less than $5 \%$ are commonly used. In addition, buffer concentrations more than $50 \mathrm{mM}$ lead to salt deposition at the nebulizer tip and $\mathrm{MS}$ sampling cone. In conclusion, LC-ICP-MS has the analytical performances needed for the speciation of arsenic in clinical chemistry but is expensive.

\section{KEY-WORDS}

Arsenic, speciation, LC-ICP-MS.

Tableau I : Caractéristiques des espèces d'arsenic étudiées.

\begin{tabular}{|c|c|c|c|}
\hline Nom & Formule* & $\begin{array}{l}\text { LD50*** } \\
(\mathrm{mg} / \mathrm{kg})\end{array}$ & pKa \\
\hline $\begin{array}{l}\text { Arsénite } \\
\left(\mathrm{As}^{\mathrm{III}}\right)\end{array}$ & - $\mathrm{OH}$ & 4,5 & 9.3 \\
\hline $\begin{array}{l}\text { Arséniate } \\
\left(\mathrm{As}^{\mathrm{v}}\right)\end{array}$ & - & $14-18$ & $\begin{array}{c}2.3 \\
6.9 \\
11.4\end{array}$ \\
\hline $\begin{array}{l}\text { Acide } \\
\text { monométhylarsonique } \\
\left(\mathrm{MMA}^{\mathrm{v}}\right)\end{array}$ & 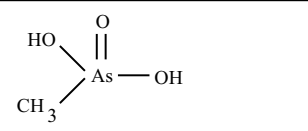 & 1800 & $\begin{array}{l}3.6 \\
8.2\end{array}$ \\
\hline $\begin{array}{l}\text { Acide } \\
\text { diméthylarsénique } \\
\left(\mathrm{DMA}^{\mathrm{v}}\right)\end{array}$ & ${ }_{\mathrm{CH}_{3}}^{\mathrm{CH}_{3}}{ }_{\mathrm{As}}^{\mathrm{O}}-\mathrm{OH}$ & 1200 & 6.2 \\
\hline $\begin{array}{l}\text { Arsénobétaïne } \\
\text { (AsB) }\end{array}$ & $\left.{ }_{\mathrm{CH}_{3}}^{\mathrm{CH}_{3}}\right|_{\mathrm{As}^{+}-} ^{\mathrm{CH}_{3}}-\mathrm{CH}_{2}-\mathrm{COOH}$ & 10000 & 4.7 \\
\hline
\end{tabular}

France, la valeur limite pour la concentration d'arsenic dans l'eau destinée à la consommation humaine est de $10 \mu \mathrm{g} / \mathrm{L})$. L'acide diméthylarsinique $\left(\mathrm{DMA}^{\mathrm{V}}\right)$ sous forme de sel de sodium (cacodylate) est commercialisé comme anti-fourmi mais l'ingestion de ce produit, souvent par des enfants, est généralement sans danger. Dans la population générale, l'exposition alimentaire est la principale source d'arsenic. Elle provient en grande partie des produits de la mer (poissons, crustacés, mollusques). L'étude CALIPSO (3) réalisée en France, rapporte une teneur en arsenic total (Ast) 
dans les poissons comprise entre 0,7 et $34 \mu \mathrm{g} / \mathrm{g}$ de poids brut selon les espèces, avec un pourcentage en arsenic inorganique compris entre 0,1 et $3,5 \%$. Quant à l'arsénobétaïne, il représente 80 à $95 \%$ de l'arsenic présent dans les produits de la mer $(3,4,5)$. Toujours dans l'étude CALIPSO, la concentration d'arsenic total a été mesurée dans l'urine de 996 sujets consommant des produits de la mer plus de deux fois par semaine. Chez 101 sujets, la concentration urinaire en Ast était supérieure à $75 \mu \mathrm{g} / \mathrm{g}$ créatinine $(\mathrm{m}=259 \pm 331$, valeur maximale : $3050 \mu \mathrm{g} / \mathrm{g}$ créatinine). Après élimination de l'arsénobétaïne par une méthode d'extraction liquideliquide (6), la concentration moyenne en arsenic était égale à $26,5 \pm 22,3 \mu \mathrm{g} / \mathrm{g}$ créatinine. Torra et coll. (7) ont montré par ailleurs que dans une population vivant à Barcelone, $79 \%$ de l'arsenic excrété dans les urines provenait de la consommation de produits de la mer.

Il est évident que le seul dosage de l'arsenic total ne permet pas une interprétation correcte en biologie médicale que ce soit dans un contexte médico-légal ou de surveillance d'une exposition professionnelle. Il peut être utilisé comme analyse de dépistage pour écarter une éventuelle intoxication. La spéciation s'impose en présence d'un dosage d'arsenic total élevé.

De nombreuses techniques permettent aujourd'hui de séparer et de quantifier les différentes formes chimiques de l'arsenic et plusieurs articles de type revue leur sont consacrés $(4,8)$. Le couplage de la chromatographie en phase liquide à la spectrométrie de masse avec torche à plasma (LC-ICP-MS) est l'une des plus utilisées.

Le but de cet article est de présenter brièvement les avantages et les inconvénients de la LC-ICP-MS en biologie médicale.

\section{Avantages de la LC-ICP-MS Sensibilité}

La spéciation de l'arsenic dans les milieux biologiques requiert une technique de détection très sensible si l'on veut atteindre les niveaux de concentration rencontrés chez les sujets non exposés. En effet, celles-ci sont de l'ordre du $\mu \mathrm{g} / \mathrm{L}$ dans le sang et l'urine (mis à part l'arsenic provenant de l'arsénobétaïne dont la concentration peut dépasser le $\mathrm{mg} / \mathrm{L}$ dans l'urine après consommation de produits de la mer).

Dans le sang, la concentration d'arsenic total chez les sujets non exposés est généralement inférieure à $10 \mu \mathrm{g} / \mathrm{L}$ (9). Mandal et coll. (10) ont trouvé une concentration moyenne de $2,25 \mu \mathrm{g} / \mathrm{L}$ chez 15 sujets témoin et Goullé et coll. (11) une valeur médiane de $5,0 \mu \mathrm{g} / \mathrm{L}\left(5^{\text {ème_}}-95^{\text {ème }}\right.$ percentile : 2,6-17,9 $\left.\mu \mathrm{g} / \mathrm{L}\right)$ chez 100 sujets témoin.

Dans l'urine, la concentration d'arsenic d'origine minérale $\left(\mathrm{As}^{\mathrm{III}}+\mathrm{As}^{\mathrm{V}}+\mathrm{DMA}^{\mathrm{V}}+\mathrm{MMA}^{\mathrm{v}}\right)$ est inférieure à $10 \mu \mathrm{g} / \mathrm{g}$ créatinine dans la population générale (9). La spéciation de l'arsenic dans l'urine a surtout été étudiée dans des populations exposées chez lesquelles une répartition moyenne de l'ordre de 10-20\% (Asi), 10-20\% (MMA $\left.{ }^{\mathrm{V}}\right)$ et $60-80 \%\left(\mathrm{DMA}^{\mathrm{V}}\right)$ est observée (12). Les données concernant la spéciation de l'arsenic dans l'urine de sujets non exposés sont rares. Dans le tableau II sont rassemblées les concentrations urinaires de $A s^{\mathrm{III}}, \mathrm{As}^{\mathrm{v}}, \mathrm{MMA}^{\mathrm{V}}$ et $\mathrm{DMA}^{\mathrm{v}}$ mesurées par différents auteurs chez des sujets témoin $(4,10,13,14)$.

Tableau II : Concentration (en $\mu g / L$ d'arsenic) de $A s^{I I I}, A s^{V}, D M A^{V}$, $M M A^{V}$ dans l'urine de sujets non exposés.

\begin{tabular}{|c|c|c|c|c|c|}
\hline & $\begin{array}{c}\mathbf{A s}^{\mathrm{III}} \\
(\mu \mathrm{g} / \mathrm{L})\end{array}$ & $\underset{(\mu \mathrm{g} / \mathbf{L})}{\mathbf{A s}^{\mathrm{V}}}$ & $\begin{array}{l}\mathrm{DMA}^{\mathrm{v}} \\
(\mu \mathrm{g} / \mathrm{L})\end{array}$ & $\begin{array}{l}\mathrm{MMA}^{\mathrm{V}} \\
(\mu \mathrm{g} / \mathrm{L})\end{array}$ & $\begin{array}{l}\text { Réfé- } \\
\text { rences }\end{array}$ \\
\hline $\begin{array}{c}\text { moyenne } \\
(n=101)\end{array}$ & $\begin{array}{c}<1(\mathrm{ld}) \\
{[11,9 \%]^{*}}\end{array}$ & $\begin{array}{c}<10(\mathrm{ld}) \\
{[0 \%]^{*}}\end{array}$ & $\begin{array}{c}10,5 \\
{[88,1 \%]^{*}}\end{array}$ & $\begin{array}{l}<2(\mathrm{ld}) \\
{[0 \%]^{*}}\end{array}$ & 14 \\
\hline $\begin{array}{c}\text { moyenne } \\
(\mathrm{n}=34)\end{array}$ & $\begin{array}{l}<0,8(\mathrm{ld}) \\
{[26,5 \%]^{*}}\end{array}$ & $\begin{array}{l}<0,8(\mathrm{ld}) \\
{[26,5 \%]^{*}}\end{array}$ & $\begin{array}{c}4,6 \\
{[100 \%]^{*}}\end{array}$ & $\begin{array}{c}0,7 \\
{[100 \%]^{*}}\end{array}$ & 13 \\
\hline $\begin{array}{c}\text { moyenne } \\
(\mathrm{n}=15)\end{array}$ & $<0,14$ (ld) & $<0,33$ (ld) & $6,49 \pm 4,05$ & $3,12 \pm 1,75$ & 10 \\
\hline $\begin{array}{c}\text { moyenne } \\
(\mathrm{n}=148)\end{array}$ & \multicolumn{2}{|c|}{$\mathrm{As}^{\mathrm{III}}+\mathrm{As}^{\mathrm{V}}: 1,9$} & 2,1 & 1,9 & 4 \\
\hline $\mathrm{ld}=\lim$ & e détec & 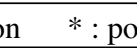 & age & hantillon & ositifs \\
\hline
\end{tabular}

Dans les cheveux, des concentrations d'arsenic total inférieures à $1 \mu \mathrm{g} / \mathrm{g}$ sont généralement considérées comme non toxiques. Mandal et coll. (15) ont trouvé des concentrations moyennes d'arsenic total de $0,07 \mu \mathrm{g} / \mathrm{g}(0,03$ à $0,12 \mu \mathrm{g} / \mathrm{g})$ dans les cheveux et de $0,19 \mu \mathrm{g} / \mathrm{g}(0,11$ à $0,30 \mu \mathrm{g} / \mathrm{g})$ dans les ongles de 15 sujets témoin. Goullé et coll. (11) rapportent une valeur médiane d'arsenic total de $0,05 \mu \mathrm{g} / \mathrm{g}\left(5^{\text {ème }}-95^{\text {ème }}\right.$ percentile : $0,03-0,08 \mu \mathrm{g} / \mathrm{g}$ ) dans les cheveux de 45 sujets témoin. L'arsenic dans les phanères est majoritairement sous forme inorganique, $\mathrm{As}{ }^{\mathrm{III}}+\mathrm{As}^{\mathrm{V}}$ représentant environ $85 \%$ de l'arsenic total $(10,15,16)$. A l'inverse de ce qui est observé dans l'urine, une forte consommation de produits marins n'influe pas sur la concentration en arsenic dans le cheveu, l'arsénobétaïne n'étant pas fixé dans les cheveux $(15,16)$. Le problème majeur rencontré lors du dosage de l'arsenic dans le cheveu n'est pas la spéciation mais la contamination externe. L'arsenic exogène se fixe de façon irréversible et aucune méthode de lavage efficace permet de l'éliminer (17). Il est donc impossible de différencier l'arsenic d'origine exogène de l'arsenic d'origine endogène.

La spectrométrie de masse couplée à un plasma induit par haute fréquence permet d'accéder à des limites de détection compatibles avec la spéciation de l'arsenic dans les milieux biologiques. Elle présente comme avantage supplémentaire une très large gamme dynamique 
linéaire. Les limites de détection dans l'urine, obtenues par LC-ICP-MS, varient selon les auteurs et les espèces d'arsenic de 0,5 $\mu \mathrm{g} / \mathrm{L}$ à $10 \mu \mathrm{g} / \mathrm{L}$.

\section{Spécificité}

L'arsenic ne possède qu'un seul isotope naturel et ne peut donc être détecté que par l'ion m/z 75. L'interférence polyatomique ${ }^{40} \mathrm{Ar}^{35} \mathrm{Cl}^{+}$formée par combinaison $\mathrm{du}$ chlorure présent dans les échantillons biologiques avec l'argon du plasma est la principale limitation au dosage de l'arsenic par ICP-MS. Le couplage à la LC permet de s'affranchir de cette interférence car l'ion chlorure peut aisément être séparé des espèces arséniées par chromatographie échangeuses d'ions ou d'appariement d'ions.

\section{Identification de composés inconnus}

La LC-ICP-MS peut être utilisée comme technique de screening pour mettre en évidence la présence d'espèces d'arsenic dans des échantillons biologiques (18). Ces composés pourront ensuite être identifiés par comparaison des temps de rétention avec des standards ou par spectrométrie de masse moléculaire dans le cas de nouvelles espèces arséniées (8).

\section{Facilité du couplage LC et ICP-MS}

La mise en œuvre du couplage entre le système chromatographique et l'ICP-MS est extrêmement simple. Il suffit de raccorder la sortie de la colonne par un tube (type tube en PEEK ${ }^{\circledR}$ ) au nébuliseur de l'ICP. En effet, les débits classiquement utilisés en chromatographie liquide $(\approx 1 \mathrm{ml} / \mathrm{min})$ sont compatibles avec le fonctionnement de la torche à plasma.

\section{Inconvénients de la LC-ICP-MS \\ Coût}

Le coût d'investissement et de fonctionnement d'un ICP-MS n'est pas à la portée de tous les laboratoires de biologie médicale. Le coût de fonctionnement est essentiellement lié à la consommation d'argon $(15 \mathrm{~L} / \mathrm{min})$. C'est un paramètre à prendre en compte en LC-ICPMS car si la durée moyenne d'une analyse en mode de fonctionnement ICP-MS normal est d'environ deux minutes, la durée de l'analyse chromatographique est plutôt de l'ordre d'une dizaine de minutes voire plus. Il est bien évident que par mesure d'économie, il est recommandé d'équilibrer la colonne en off-line c'est-àdire avant de la connecter à l'ICP-MS.

\section{Analyse chromatographique}

La qualité de la séparation chromatographique est primordiale car les différentes espèces chimiques de l'arsenic sont identifiées sur la base de leur temps de rétention. Or, le couplage de la LC à l'ICP-MS impose des contraintes concernant la composition de la phase mobile. Une concentration trop élevée en solvant organique déstabilise le plasma et peut l'éteindre aussi des concentrations inférieures à 5\% sont habituellement utilisées. D'autre part, des concentrations de tampon supérieures à $50 \mathrm{mM}$ peuvent être à l'origine de dépôts de sels au niveau du nébuliseur et du cône échantillonneur. Une autre difficulté réside dans le fait que l'arsénobétaïne dans l'urine se trouve souvent en quantité beaucoup plus importante que les autres espèces arséniées et doit donc être bien résolu chromatographiquement. La séparation sur une même colonne des cinq espèces d'arsenic les plus étudiées, $\mathrm{As}^{\mathrm{III}}, \mathrm{As}^{\mathrm{v}}, \mathrm{DMA}^{\mathrm{v}}, \mathrm{MMA}^{\mathrm{v}}$ et $\mathrm{AsB}$ reste délicate.

Les propriétés physico-chimiques et en premier lieu les pKa des formes chimiques de l'arsenic (Tableau I) (19) permettent une séparation par différents types de chromatographie : échange d'anions, de cations ou phase inverse avec appariement d'ions.

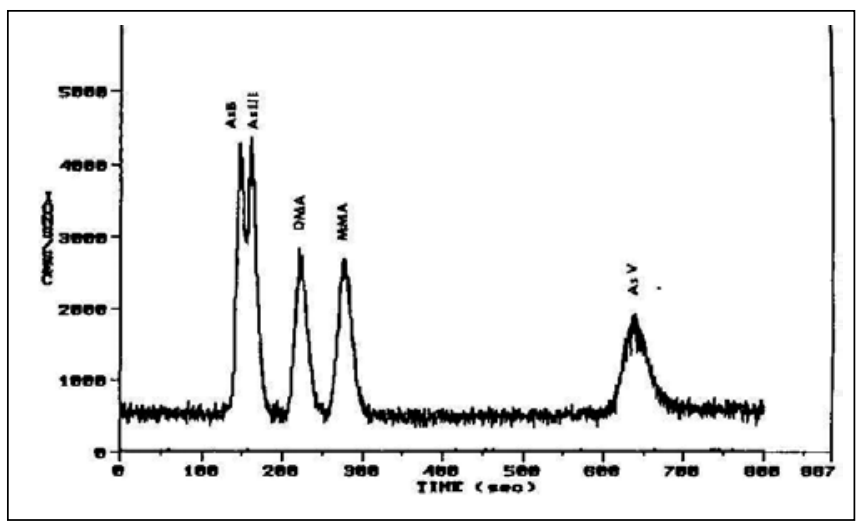

Figure 1 : Séparation de 5 composés arséniés (solution standard à $100 \mu \mathrm{g} / \mathrm{L}$ d'arsenic). Conditions chromatographiques : colonne PRP-X100, phase mobile : tampon phosphate 22,5 mM (pH 6,2), $1 \mathrm{~mL} / \mathrm{min}$.

La chromatographie par échange d'anions est le mode de séparation le plus utilisé avec notamment la colonne PRP-X100 de chez Hamilton. La difficulté en échange d'anions est de séparer AsB et As ${ }^{\text {III. }}$. Pour des valeurs de $\mathrm{pH}$ comprises entre 2 et 8 , AsB et $\mathrm{As}^{\mathrm{III}}$ ne sont pas retenus sur la colonne, $\mathrm{As}{ }^{\mathrm{III}}$ n'étant pas ionisé et AsB étant sous forme de zwittérion ou de cation selon le $\mathrm{pH}$. Ils sont coélués en début de colonne alors que les autres espèces, $\mathrm{As}^{\mathrm{v}}, \mathrm{DMA}^{\mathrm{v}}$ et $\mathrm{MMA}^{\mathrm{v}}$, sous forme d'anions, sont retenues et bien séparées. La figure 1 montre un exemple de chromatogramme obtenu dans notre laboratoire à $\mathrm{pH}$ 6,2 . En augmentant le $\mathrm{pH}$ de la phase mobile jusqu'à des $\mathrm{pH}>9$, $\mathrm{As}^{\mathrm{III}}$ s'ionise et est donc mieux retenu sur la colonne. Ceci suppose des colonnes à support polymérique, ce qui est le cas de la PRP-X100. Cette augmentation du $\mathrm{pH}$ s'accompagne d'un allongement du temps de rétention de As ${ }^{\mathrm{v}}$. Ainsi, Morton et coll. (13) 
obtiennent une séparation des cinq espèces d'arsenic (AsB, $\mathrm{As}^{\mathrm{III}}, \mathrm{As}^{\mathrm{v}}, \mathrm{DMA}^{\mathrm{V}}$ et $\mathrm{MMA}^{\mathrm{V}}$ ) sur la colonne PRP-X100 en gradient de solvant $(10 \mathrm{mM}$ à $20 \mathrm{mM}$ de carbonate d'ammonium) mais ceci aux dépends d'un temps d'analyse de $27 \mathrm{~min}$. Des séparations similaires peuvent être obtenues en chromatographie par appariement d'ions sur des colonnes de type C18 avec comme contre-ion des sels d'ammonium quaternaire (5, 20, 21).

La chromatographie par échange de cations permet de retenir AsB qui est alors élué en dernier mais $\mathrm{As}^{\mathrm{III}}$ et $\mathrm{As}^{\mathrm{V}}$ sont coélués $(16,20,22,23)$. La chromatographie en phase inverse par appariement d'ions avec des sulfonates comme contre-ion donne des résultats très proches (24).

Une revue des différentes méthodes de séparation des espèces arséniées en chromatographie liquide été réalisée par Benramdane et coll. (4).

\section{Disponibilité de l'appareil}

Le couplage LC-ICP-MS est une technique peu adaptée aux dosages ponctuels mais plutôt aux dosages en série. Peu de laboratoires disposent de plusieurs ICP-MS or l'utilisation en mode de couplage LC-ICP-MS mobilise l'appareil pour des durées importantes, le rendant indisponible pour les analyses classiques. Pour les laboratoires, ayant la possibilité et une forte demande d'analyses dans ce domaine, il est préférable de dédier un ICP-MS au couplage avec la LC.

\section{Disponibilité des "standards" et des matériaux de référence}

Si pour l'analyse de l'arsenic inorganique, les standards sous forme de solutions (généralement à $1 \mathrm{~g} / \mathrm{L}$ ) sont largement distribués, il n'en est pas de même pour l'analyse des composés organiques. Beaucoup de ces composés, même sous forme de produit pur, ne sont pas commercialisés. Quant aux matériaux de référence disponibles (sang, urine, cheveu), ils ne sont certifiés que pour leur teneur en arsenic total et donc peu adaptés à la spéciation. Le développement des méthodes et l'augmentation conjointe de la demande dans le domaine de la spéciation amèneront vraisemblablement à la commercialisation de matériaux certifiés appropriés. Ceci est déjà le cas pour la spéciation du mercure, de l'étain et de l'arsenic dans les produits de la mer.

\section{Stabilité des espèces chimiques d'arsenic}

La stabilité du degré d'oxydation des formes d'arsenic est un problème en spéciation. Les dérivés pentavalents sont beaucoup plus stables que leurs homologues trivalents. Une oxydation peut se produire au cours du stockage des échantillons ou pendant l'analyse. Comme Larsen et coll. (25), nous avons observé une transformation partielle de $\mathrm{As}^{\mathrm{III}}$ en $\mathrm{As}^{\mathrm{V}}$ au cours de l'analyse chromatographique. Par ailleurs, des études récentes $(2,10,26)$ ont mis en évidence la présence de $\mathrm{MMA}^{\mathrm{III}}$ et de $\mathrm{DMA}^{\mathrm{III}}$ dans les urines de sujets chroniquement exposés par une eau contaminée. Ces dérivés méthylés trivalents qui auraient une toxicité supérieure à celle des dérivés inorganiques $\mathrm{As}^{\mathrm{III}}$ et $\mathrm{As}^{\mathrm{V}}(27,28)$ sont cependant très peu stables en solution et s'oxydent rapidement en leurs homologues pentavalents. Del Razo et coll. (26) ont montré que dans l'urine conservée à $4^{\circ} \mathrm{C}, 14-36 \%$ de $\mathrm{MMA}^{\mathrm{III}}$ et $17-79 \%$ de DMA ${ }^{\mathrm{III}}$ étaient oxydés après 24h, 24-43\% de MMA ${ }^{\text {III }}$ et 33-86\% de DMA ${ }^{\mathrm{III}}$ après une semaine et $30-100 \%$ de $\mathrm{MMA}^{\mathrm{III}}$ et $42-100 \%$ de $\mathrm{DMA}^{\mathrm{III}}$ au bout de deux mois. Le et coll. (2) ont également observé que dans l'urine environ $60 \%$ de $\mathrm{MMA}^{\mathrm{III}}$ et 95\% de $\mathrm{DMA}^{\mathrm{III}}$ étaient transformés respectivement en $\mathrm{MMA}^{\mathrm{v}}$ et $\mathrm{DMA}^{\mathrm{v}}$ après deux semaines de conservation à $4^{\circ} \mathrm{C}$.

Feldmann et coll. (29) ont montré que les cinq espèces d'arsenic les plus couramment dosées, $\mathrm{As}^{\mathrm{III}}, \mathrm{As}^{\mathrm{v}}$, AsB, $\mathrm{DMA}^{\mathrm{v}}$ et $\mathrm{MMA}^{\mathrm{v}}$ étaient stables dans l'urine pendant deux mois à 4 et à $-20^{\circ} \mathrm{C}$.

\section{Conclusion}

En conclusion, le couplage LC-ICP-MS est une technique performante pour la spéciation de l'arsenic dans les milieux biologiques mais coûteuse et peu adaptée à des dosages ponctuels. Le problème de la stabilité du degré d'oxydation des espèces d'arsenic dans les milieux biologiques ainsi que la disponibilité des produits purs et des matériaux de référence se pose indépendamment de la technique d'analyse utilisée.

\section{Références}

1. Anger J.P., Labat L., Lhermitte M. La biométhylation des métaux chez l'homme et dans l'environnement: succès ou échec sur le plan toxicologique ? Ann. Toxicol. Anal. $2005 ; 17$ (3) : 175-86.

2. Le X. C., Lu X., Ma M., Cullen W.R., Aposhian H. V., Zheng B. Speciation of key arsenic metabolic intermediates in human urine. Anal. Chem. 2000 ; 72 : 5172-7.

3. AFSSA. Leblanc J.C. (coordonnateur). CALIPSO : Etude des consommations alimentaires de produits de la mer et imprégnation aux éléments traces, polluants et oméga 3. 2006.

4. Benramdane L., Bressolle F., Vallon J.J.Arsenic speciation in humans and food products : a review. J. Chromatogr. Sci. $1999 ; 37: 330-44$.

5. Thomas P., Sniatecki K. Inductively coupled plasma mass spectrometry : application to the determination of arsenic species. Fresenius J. Anal. Chem. 1995 ; 351 : 410-4.

6. Le Bouil A., Notelet S., Cailleux A., Turcant A., Allain P. Dosage de l'arsenic urinaire après séparation de l'arsénobétaïne. Toxicorama. 1997 ; IX (3) : 171-6. 
7. Torra M., Augé J.M., Ballell B., Rodamilans M., Casoliva C., Corbella J. Biological monitoring of environmental exposure to arsenic in urine samples from residents of the city of Barcelona, Spain, after selective extraction of the toxicologically relevant arsenic forms. Trace Elem. Elec. 2004 ; 21 (4) : 248-51.

8. Francesconi K.A., Kuehnelt D. Determination of arsenic species : A critical review of methods and applications, 200-2003. Analyst. $2004 ; 129$ : 373-95

9. INRS. Biotox. Arsenic. 2002 ; p. 71-2.

10. Mandal B.K., Ogra Y., Anzai K., Suzuki K.T. Speciation of arsenic in biological samples. Toxicol. Appl. Pharmacol. 2004 ; $198: 307-18$

11. Goullé J.P., Mahieu L., Castermant J., Neveu N., Bonneau L., Lainé G., Bouige D., Lacroix C. Metal and metalloid multi-elementary ICP-MS validation in whole blood, plasma, urine and hair References values. Forensic Sci. Int. $2005 ; 153: 39-44$.

12. VahterM.,Genetic polymorphism in the biotransformation of inorganic arsenic and its role in toxicity. Toxicol. Lett. 2002 ; 112-113 : 209-17.

13. Morton J., Mason H. Speciation of arsenic compounds in urine from occupationally unexposed and exposed persons in the U.K. using a routine LC-ICP-MS method. J. Anal. Toxicol. $2006 ; 30: 293-301$.

14. Heinrich-Ramm R., Mindt-Prüfert S., Szadkowski D. Arsenic species in a group of persons in northern Germany - Contribution to the evaluation of reference values. Int. J. Hyg. Environ. Health. 2001 ; 203 : 475-77.

15. Mandal B. K., Ogra Y., Suzuki K.T. Speciation of arsenic in human nail and hair from arsenic-affected area by HPLC - inductively coupled argon mass spectrometry. Toxicol. Appl. Pharmacol. 2003 ; 189 : 73-83.

16. Raab A., Feldmann J. Arsenic speciation in hair extracts. Anal. Bioanal. Chem. 2005 ; 381 : 332-8.

17. Morton J., Carolan V.A., Gardiner P.H.E. Removal of exogenously bound elements from human hair by various washing procedures and determination by inductively coupled plasma mass spectrometry. Anal. Chim. Acta. $2002 ; 455: 23-34$.

18. Szpunar J. Trace element speciation analysis of biomaterials by high-performance liquid chromatography with inductively coupled plasma mass spectrometry detection. Trends Anal. Chem. 2000 ; 19 : 127-37.

19. Albert M., Demesnay C., Porthault M., Rocca J.L. Séparation d'espèces arséniées organiques et minérales par chromatographie en phase liquide et électrophorèse capillaire. Analusis. $1992 ; 20: 383-90$.
20. Zhang X., Cornelis R., De Kimpe J., Mees L., Vanderbiesen V., De Cubber A., Vanholder R. Accumulation of arsenic species in serum of patients with chronic renal disease. Clin. Chem. 1996 ; 42 : 1231-7.

21. Shraim A., Cui X., Li S., Ng J.C., Wang J., Jin Y., Liu Y., Guo L., Li D., Wang S., Zhang R., Hirano S. Arsenic speciation in the urine and hair of individuals exposed to airbone arsenic through coal-burning in Guizhou, PR China. Toxicol. Lett. 2003 ; 137 : 35-48.

22. Morita M., Uehiro T., Fuwa K. Determination of arsenic compounds in biological samples by liquid chromatography with inductively coupled argon plasmaatomic emission spectrometric detection. Anal. Chem. $1981 ; 53: 1806-8$.

23. Simon S., Lobos G., Pannier F., De Gregori I., Pinochet H., Potin-Gautier M. Speciation analysis of organoarsenical in biological matrices by coupling ion chromatography to atomic fluorescence spectrometry with on-line photooxidation and hydride generation. Anal. Chim. Acta. 2004 ; 521 : 99-108.

24. Le X.C., Ma M. Speciation of arsenic compounds by using ion-pair chromatography with atomic spectrometry and mass spectrometry detection. J. Chromatogr. A. 1997 ; 764 : 55-64.

25. Larsen E. H., Pritzl G., Hansen S. H. Speciation of eight arsenic compounds in human urine by high-performance liquid chromatography with inductively coupled plasma mass spectrometric detection using antimonate for internal chromatographic standard. J. Anal. Atomic. Spectrom. $1993 ; 8: 557-63$.

26. Del Razo L.M., Styblo M., Cullen W.R., Thomas D.J. Determination of trivalent methylated arsenicals in biological matrices. Toxicol. Appl. Pharmacol. 2001 ; 174 : 282-93.

27. Styblo M., Del Razo L. M., Vega L., Germolec D.R., LeCluyse E.L., Hamilton G.A., Reed W., Wang C., Cullen W.R., Thomas D.J. Comparative toxicity of trivalent and pentavalent inorganic and methylated arsenicals in rat and human cells. Arch. Toxicol. 2000 ; 74 : 289-99.

28. Mass M.J., TennantA., Roop B.C., Cullen W.R., Styblo M., Thomas D.J., Kligerman A.D. Methylated trivalent arsenic species are genotoxic. Chem. Res. Toxicol. $2001 ; 14: 355-61$.

29. Feldmann J., Lai V.W-M., Cullen W.R., Ma M., Lu X., Le X.C. Sample preparation and storage can change arsenic speciation in human urine. Clin. Chem. 1999 ; 45 : 198897. 